\title{
Lucky Imaging Survey for Binary Exoplanet Hosts
}

\author{
Carolina Bergfors ${ }^{1}$, Wolfgang Brandner ${ }^{1}$, Sebastian Daemgen ${ }^{2}$ \\ and Thomas Henning ${ }^{1}$ \\ ${ }^{1}$ Max-Planck-Institut für Astronomie, Königstuhl 17, \\ 69117 Heidelberg, Germany \\ email: bergfors@mpia.de \\ ${ }^{2}$ European Southern Observatory, Karl-Schwarzschild-Strasse 2, \\ 85748 Garching, Germany
}

\begin{abstract}
Binary or multiple stars are common in our neighbourhood, and many of the exoplanets we know of belong to a star in such a system. The influence of a second star on planet formation can be probed by comparing properties of planets in binary/multiple-star systems with those of single-star planets. We present some of the results from our Lucky Imaging survey for binary companions to hosts of transiting exoplanets.
\end{abstract}

Keywords. Techniques: high angular resolution - Binaries: visual - Planetary systems

\section{Introduction}

The presence of a close stellar companion is expected to affect the formation of planets. System characteristics such as the occurrence of a stellar companion and the binary orbital separation together with planetary properties provide valuable constraints on models of planet formation and dynamical evolution.

Exoplanets that transit their parent star can provide essential properties such as radius and mass, from which the mean density and surface gravity can be derived. Previous large studies of the physical properties of planets in binary star systems have primarily included exoplanets found with radial velocity spectroscopy, since the number of transiting exoplanets (TEP's) has been small until lately. Almost half of the known TEP's have been found during the last year, mainly thanks to large ground- and space-based programs. The increasingly large number of transiting exoplanets allows for this study of how binarity/multiplicity affects their properties.

\section{The AstraLux survey for binary TEP hosts}

We searched for faint, close stellar companions to 21 known TEP host stars using the two Lucky Imaging instruments AstraLux Norte at the $2.2 \mathrm{~m}$ telescope at Calar Alto, Spain, for the northern sky targets, and AstraLux Sur mounted at the ESO $3.5 \mathrm{~m}$ New Technology Telescope (NTT) at La Silla, Chile, for the southern sky TEP hosts.

Two previously unknown companion candidates were discovered at $\sim 1$ arcsec separation to the TEP host stars HAT-P-8 and Wasp-12 (Bergfors et al. 2011, in prep.). We could also confirm the common proper motion of the candidate binary exoplanet host TrES-4 which was discovered earlier within this survey (Daemgen et al. 2009). 


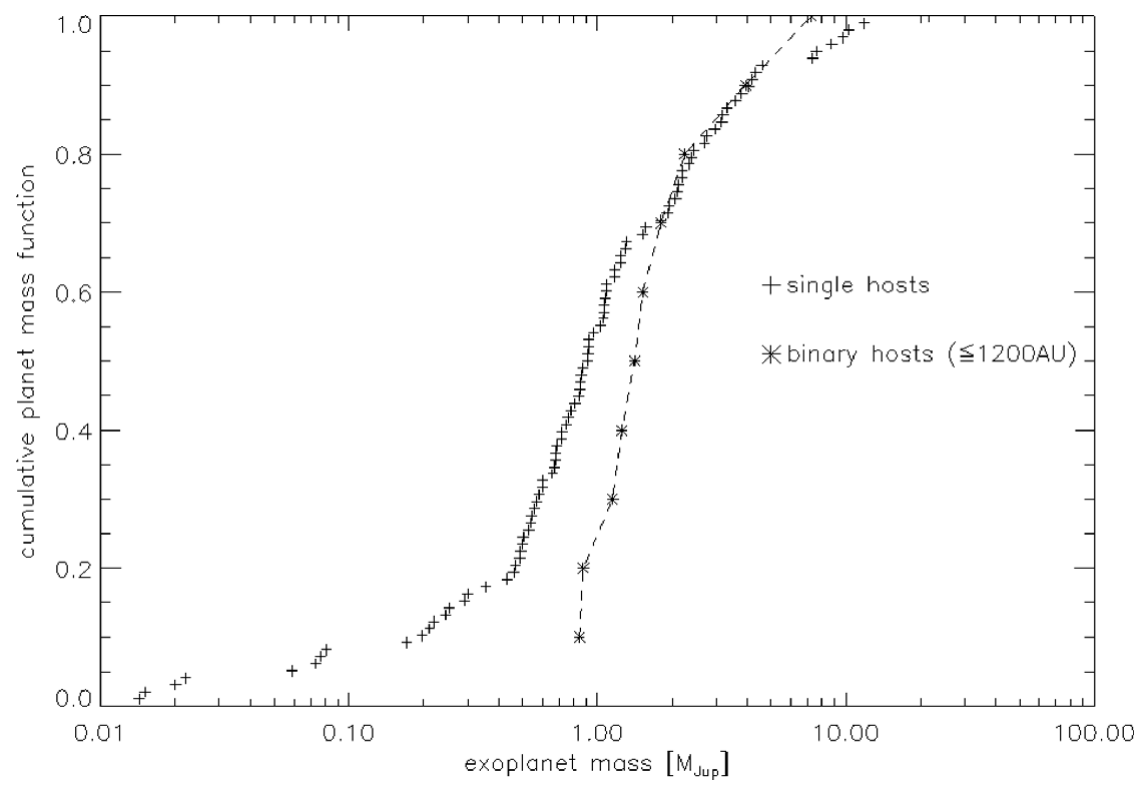

Figure 1. Cumulative planet mass function. Plus-signs represent the masses of transiting exoplanets in single-star systems, and asterisks the masses of TEP's in binary/multiple star systems closer than $1200 \mathrm{AU}$. Almost half of the planets in single-star systems have masses smaller than the lowest-mass planet in the binary/multiple star sample, indicating that a stellar companion might influence the formation of the planets.

While the sample of close binary TEP hosts is still very small, we found that

- The distributions of planetary radii, orbital periods and surface gravities do not differ significantly between TEP's in binary/multiple systems and those belonging to a single star.

- The division into two classes of hot Jupiters based on Safronov number, $\theta=0.5 \times$ $\left(v_{e s c} / v_{\text {orb }}\right)^{2}$, and equilibrium temperature, that was suggested by Hansen \& Barman (2007) based on the properties of a small number of hot Jupiters, can no longer be discerned for the much larger number of TEP's known today. Additionally, we can no longer see the correlation between binary separation and Safronov number suggested by Daemgen et al. (2009) in the larger sample of binaries.

- The transiting exoplanets closer than 1200 AU appear to be on average more massive than single-star TEP's. The cumulative mass function (Fig. 1) shows that almost half of the single-star TEP's have masses smaller than the least massive binary/multiple TEP. A Kolmogorov-Smirnov test shows that the hypothesis of both samples being drawn from the same parent distribution can be rejected with a significance of $\approx 96.5 \%$.

\section{References}

Daemgen, S., Hormuth, F., Brandner, W. et al., 2009, A\&SA, 498, 567

Hansen, B. M. S. \& Barman, T. 2007, ApJ, 671, 861

Hippler, S., Bergfors, C., Brandner, W. et al., 2009, The Messenger, 137, 14

Hormuth, F., Hippler, S., Brandner, W. et al., 2008, SPIE, 7014 